\title{
Real Time Implementation of Frost Beamformer for Underwater Communications
}

\section{Hsien-Sen Hung}

Associate Professor, Department of Electrical Engineering, National Taiwan Ocean University, 2 Pei-Ning Rd., Keelung, Taiwan, R.O.C, hshung@ntou66.ntou.edu.tw

Shun-Hsyung Chang

Professor, Department of Electrical Engineering, National Taiwan Ocean University, 2 Pei-Ning Rd., Keelung, Taiwan, R.O.C

Suan-Lin Chen

Graduate Student, Department of Electrical Engineering, National Taiwan Ocean University, 2 Pei-Ning Rd., Keelung, Taiwan, R.O.C.

Chi-Wen Chang

Graduate Student, Department of Electrical Engineering, National Taiwan Ocean University, 2 Pei-Ning Rd., Keelung, Taiwan, R.O.C.

Follow this and additional works at: https://jmstt.ntou.edu.tw/journal

Part of the Electrical and Computer Engineering Commons

\section{Recommended Citation}

Hung, Hsien-Sen; Chang, Shun-Hsyung; Chen, Suan-Lin; and Chang, Chi-Wen (2009) "Real Time Implementation of Frost Beamformer for Underwater Communications," Journal of Marine Science and Technology. Vol. 7: Iss. 1, Article 1.

DOI: $10.51400 / 2709-6998.2506$

Available at: https://jmstt.ntou.edu.tw/journal/vol7/iss1/1

This Research Article is brought to you for free and open access by Journal of Marine Science and Technology. It has been accepted for inclusion in Journal of Marine Science and Technology by an authorized editor of Journal of Marine Science and Technology. 


\section{Real Time Implementation of Frost Beamformer for Underwater Communications}

\section{Acknowledgements}

This work was supported by the National Science Council, Taiwan, R.O.C. under Contract NSC86-2611E-019-016. The authors would like to thank the National Science Council for the support of this work 


\title{
REAL TIME IMPLEMENTATION OF FROST BEAMFORMER FOR UNDERWATER COMMUNICATIONS
}

\author{
Hsien-Sen Hung*, Shun-Hsyung Chang**, Suan-Lin Chen*** and Chi-Wen Chang***
}

Keywords: Adaptive beamformer, Underwater communications, Fading channel, Digital signal processor.

\begin{abstract}
In this paper, we investigate the performance of Frost's linearly constrained minimum variance (LCMV) adaptive beamformer via computer simulations for QPSK signals transmitted over Rayleigh and Rician fading channels. Simulation results show that the Frost beamformer can still improve the signal-to-interference-plus-noise ratio, even over fading channels. For real-time underwater communications, a prototype system is implemented, which uses TMS320C40 digital signal processor chip for realization of the Frost beamformer. Through experiments in an acoustic trough the validity of the real-time Frost beamformer prototype system is verified, and the feasibility of its use in underwater communications is assessed.
\end{abstract}

\section{INTRODUCTION}

The establishment of a reliable communication link is of vital importance for high speed underwater communications. When acoustic waves propagate over underwater channels, they are often affected by timevaring multipaths due to reflections from sea surface or floor as well as refractions and scattering from nonuniform layered structure of the ocean, resulting in fading of the received signals. Relative motion between the transmitter and the receiver, as well as stochastic changes in the transmission medium, cause phase fluctuations. The combination of time-varing multipath and phase instabilities causes intersymbol interference (ISI) and synchronization problems, and is the main limitation of

Paper Received Jan. 13, 1999. Revised March 17, 1999. Accepted April 14, 1999. Author for Correspondence: Hsien-Sen Hung.

*Associate Professor, Department of Electrical Engineering, National Taiwan Ocean University, 2 Pei-Ning Rd., Keelung, Taiwan, R.O.C. Email:hshung@ntou66.ntou.edu.tw

**Professor, Department of Electrical Engineering, National Taiwan Ocean University, 2 Pei-Ning Rd., Keelung, Taiwan, R.O.C.

***Graduate Student, Department of Electrical Engineering, National Taiwan Ocean University, 2 Pei-Ning Rd., Keelung, Taiwan, R.O.C. an underwater acoustic channel [1]. Other adverse factors include directional interfering sources and background noise.

To overcome the problems of ISI and directional interferers, an adaptive equalizer and an adaptive beamformer are often jointly employed [2,3]. It rejects the interferers as well as the long-delayed multipaths to reduce interference level by means of adaptive beamforming and executes diversity combining on the short-delayed multipaths to increase signal level by means of adaptive equalization. As a result, the required equalizer tap length is also reduced.

The purpose of this research is twofold. First, the behavior of an adaptive beamformer over an underwater acoustic channel, which is modeled as Rayleigh or Rician fading simulator is investigated. Specifically, Frost's constrained least mean-squares algorithm [4] is adopted as the adaptive beamformer for reasons stated as follows: 1) it is one of notable adaptive broad-band beamformers developed in the 1960s and 1970s, 2) the computational complexity is relatively low among others, thus suitable for real-time implementation, and 3 ) the use of linear constraints permits flexible and extensive control over the adapted response of the beamformer. Second, this paper is devoted to real-time implementation issues concerning software beamformer realization via digital signal processor (DSP) chip. In the followings, the paper begins with a section devoted to briefly reviewing the concepts, features, and limitations of the Frost adaptive beamformer. Succeeding sections cover simulation results about the performance of the Frost beamformer over fading channels, real-time implementation issues, and experimental results. We then conclude the paper with a summary.

\section{FROST ADAPTIVE BEAMFORMER}

The basic idea behind linearly constrained minimum variance (LCMV) beamforming is to constrain the response of the beamformer so that signals from the direction of interest are passed with specified gain and phase. The weights are chosen to minimize output 
variance or power subject to the response constraint. This has the effect of preserving the desired signal while minimizing contributions to the output due to interfering signals and noise arriving from directions other than the direction of interest. Under the framework of LCMV beamforming, Frost [4] proposed the constrained least mean-squares algorithm to adjust (update) the weights. The structure of the Forst beamformer is shown in Fig. 1. When input signals are represented in complex forms, the weights $W(k)$ are adjusted according to [5]

$$
\begin{aligned}
& W(0)=F \\
& W(k+1)=P\left[W(k)-\mu x^{*}(k) y(k)\right]+F
\end{aligned}
$$

where $x(k)$ is the received signal vector at the array of sensors, $y(k)$ is the signal of the beamformer output at time $k, \mu$ is the step size for each iteration, and the superscript $*$ denotes complex conjugation. In addition, $F$ and $P$ are defined as follows.

$$
F=C\left(C^{H} C\right)^{-1} f
$$

and

$$
P=I-C\left(C^{H} C\right)^{-1} C^{H}
$$

where the superscript $H$ denotes the Hermitian transpose. The constraint matrix $C$ is comprised of the direction vectors at each frequency, steered to the direction of the desired signal. The vector $f$ is usually chosen to have all one's in its entities for the desired signal to be of no distortion. To ensure convergence, the value of $\mu$ should be positive and be less than the reciprocal of the largest eigenvalue pertaining to the sample correlation

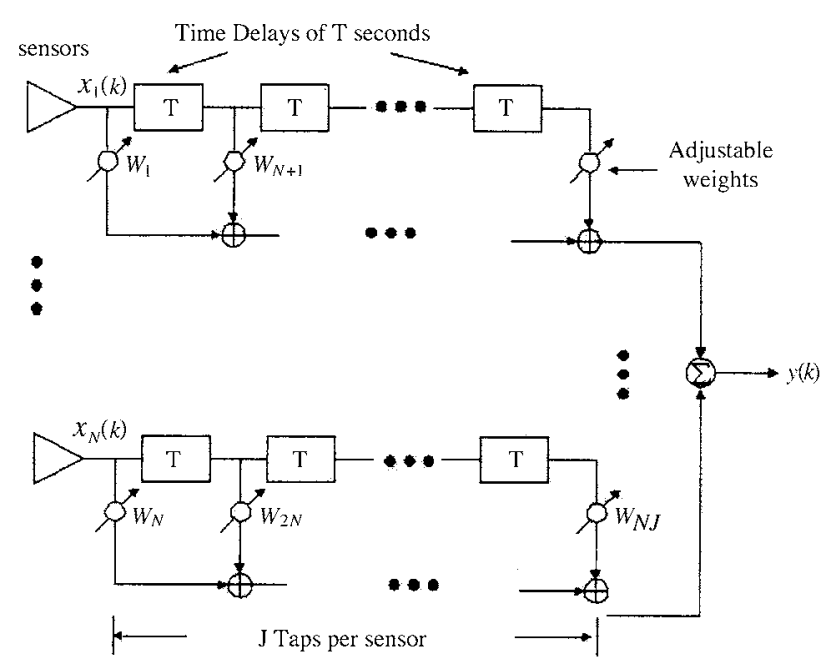

Fig. 1. The Forst beamformer's structure. matrix of the received array data vector.

The computational complexity of the beamformer algorithm is quite low, thus is very suitable for real time implementing on a single-chip DSP. However, its performance is rather sensitive to the array deformation and pointing errors, and is less effective where coherent signals caused by multipaths exist. It should be noted that the generalized sidelobe canceller (GSC) of Griffiths and Jim [6] represents an alternative implementation to LCMV beamforming. The GSC structure simplifies the LCMV beamforming implementation.

\section{PERFORMANCE OF FROST BEAMFORMER OVER FADING CHANNELS}

The model of digital data transmission over underwater acoustic channel for computer simulation is shown in Fig. 2. In this model, the transmitter comprises an encoder and a QPSK modulator. The encoder samples successive pairs of bits and generates a four-level (e.g., $00,01,10,11)$ stream of data symbols with the symbol rate of $2400 \mathrm{Bd}$. The QPSK modulator produces modulated waveforms with a phase change of $\left[-45^{\circ}, 45^{\circ}\right.$, $+135^{\circ},-135^{\circ}$ ] for each of the four levels. These modulated waveforms (as the desired signal) with a carrier frequency of $20 \mathrm{KHz}$ propagate through an underwater acoustic channel model and impinge on a sensor array with the angle of $20^{\circ}$. There also exists a sinusoidal wave of frequency $19.5 \mathrm{KHz}$ impinging upon the sensor array with $-10^{\circ}$, as the interferer. The composite signal, comprising the desired QPSK signal and the continuous wave (CW) interfering signal, is received by each sensor and further corrupted by an addtitive Gaussian white noise process, uncorrelated across each sensor.

The array consists of 4 hydrophones positioned on a line with half wavelength as its spacing between any

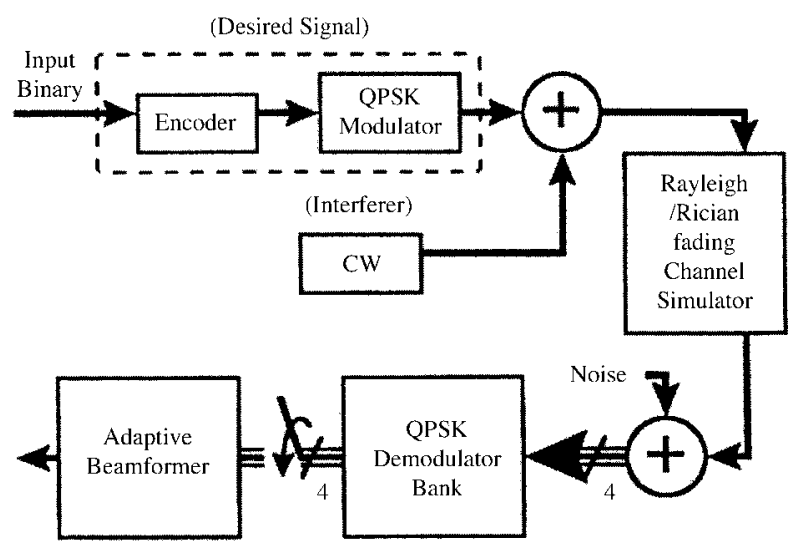

Fig. 2. Model of data transmission over underwater acoustic channel. 
two consecutive sensors. At the receiver a bank of four QPSK demodulators converts each input signal into its baseband version. The baseband signals are then sampled every bit interval and fed into the adaptive beamformer for processing. In the adaptive beamformer structure, the bit interval is chosen as the step size, and each sensor has a single tap for convenience.

In the followings, we present performance results of the Frost adaptive beamformer over an underwater acoustic channel, which is modeled as Rayleigh or Rician fading simulator. At any point in the ocean, the receiver may be located in the path of one or more rays (eigenrays), each being modeled by a single Rayleigh fading [7] or Rician fading [8] simulator.

\section{A. Performance of Frost adaptive beamformer over one- eigenray Rayleigh fading channel}

The Rayleigh fading simulator of Jakes's model [9] was used to generate envelope waveforms of Rayleigh distribution. Fig. 3(a) shows a sample waveform of Rayleigh fades with $0 \mathrm{~dB}$ as its mean value (mean=1). The histogram of Fig. 3(b) and the cumulated histogram of Fig. 3(c) for the generated envelope waveforms, reveal their closeness to the thoretical Rayleigh probability density function (PDF) and cumulative distribution function $(\mathrm{CDF})$, respectively.

The average powers for the desired signal, the interferer and noise were set to be equal, thus both the signal-to-noise ratio (SNR) and the signal-to-interference ratio (SIR) are $0 \mathrm{~dB}$. Fig. 4 shows the beampattern of the adaptive beamformer, where the power gain of the interferer $\left(\right.$ at $\left.-10^{\circ}\right)$ is reduced by $21 \mathrm{~dB}$, as compared to $0 \mathrm{~dB}$ of the desired signal (at $20^{\circ}$ ). Fig. 5(a) and 5(b) plot the constellations of the demodulated signals before and after beamforming, respectively. It is clear to see that the beamformer has better performance for symbol dis-
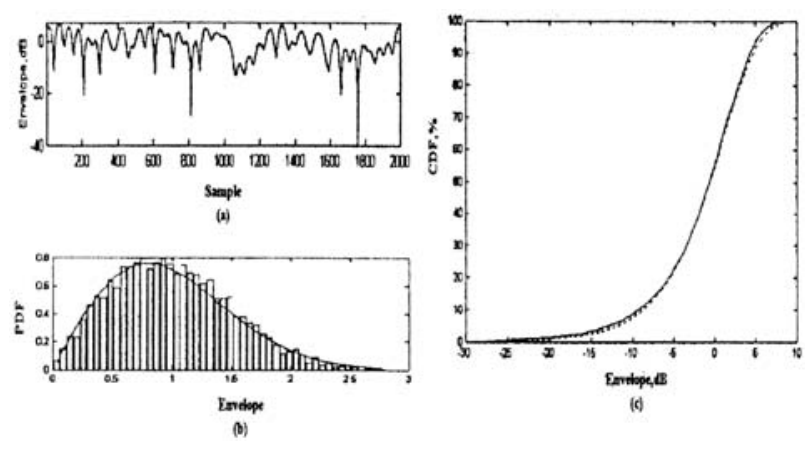

Fig. 3 (a) A sample envelope waveform of Rayleigh distribution; (b) Histogram (bar: simulation, solid line: theory) as probability density function; (c) Cumulated histogram (solid line: simulation, dotted line: theory) as cumulative distribution frunction.

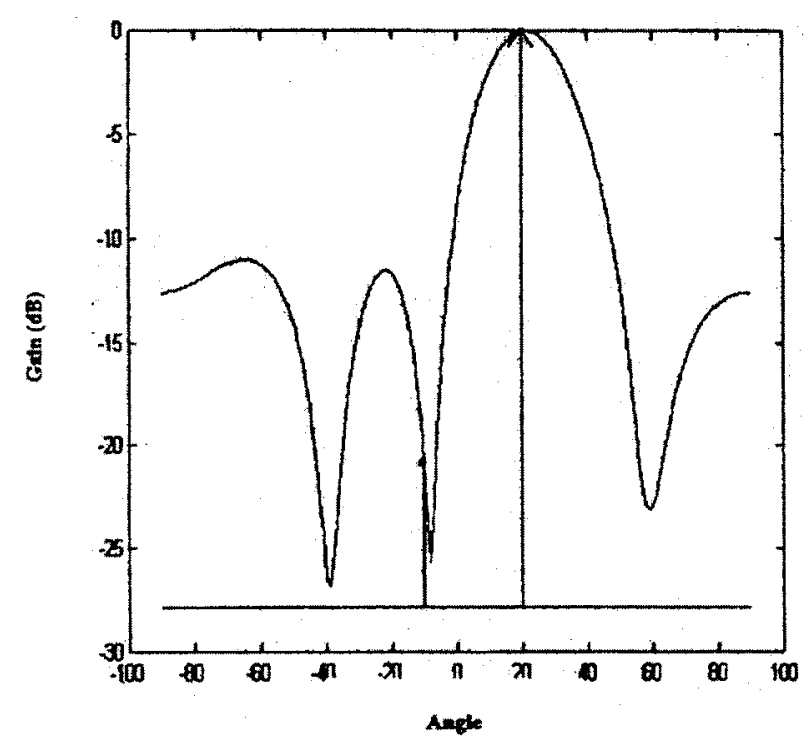

Fig. 4. Beampattern of Frost adaptive beamformer over Rayleigh fading channel.
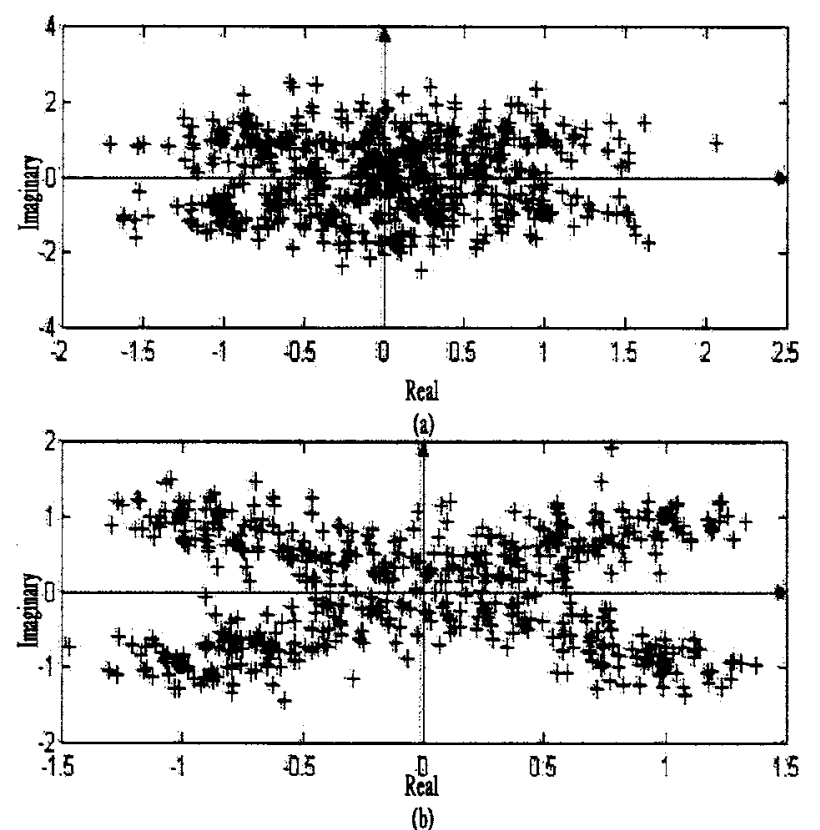

Fig. 5. Constellations of the demodulated signals, (a) before beamformer and (b) after beamformer.

crimination. In order to see the effects of the beamformer on the bit error rate (BER) for various SIRs as the SNR is fixed, thirty thousand trials were carried out. Fig. 6 compares the bit error rates before and after beamforming as a function of SIR for SNR $=0 \mathrm{~dB}$. It demonstates the efficacy of the beamformer, because the bit error rate is reduced. 


\section{B. Performance of Frost adaptive beamformer over one- eigenray Rician fading channel}

The Rician simulator is formed by adding a signal constant to the in-phase component which is generated by the Rayleigh simulator of Jakes's model. The resultant in-phase component and the quadrature component constitute a complex signal with its envelope having Rician distribution. Fig. 7(a) shows a sample Ricianfaded waveform, in which the signal constant value is 10 and the corresponding Rayleigh component has the mean value of 1. Fig. 7(b) and 7(c) show the histogram and the cumulated histogram of sample waveforms, which are very close to the theoretical Rician PDF and CDF, respectively.

Again the average powers of the desired signal, the interferer, and noise are all the same. Fig. 8 shows the beampattern of the adaptive beamformer, where the power gain of the interferer is reduced by a factor of $27 \mathrm{~dB}$, as compared to $0 \mathrm{~dB}$ of the desired signal. Fig 9 (a) and 9(b) plot the constellations of the demodulated signal before and after beamforming, respectively. It is clear that the beamformer has better performance for discriminating symbols, as evidenced by the bit error

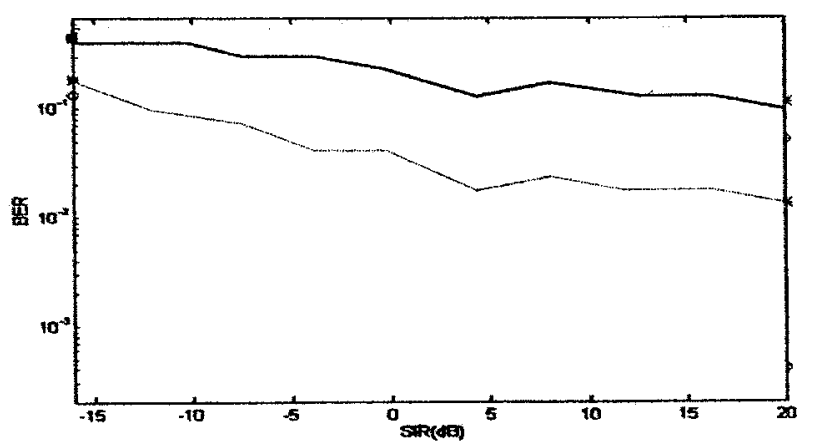

Fig. 6 Bit error rates as a function of SIR for SNR $=0 \mathrm{~dB}$; solid line: before beamformer, dotted line: after beamformer.
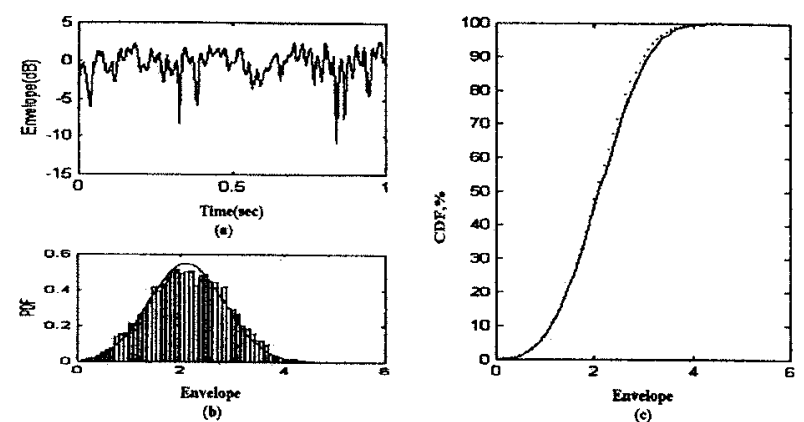

Fig. 7. (a) A sample envelope waveform of Rician distribution; (b) Histogram (bar: simulation, solid line: theory) as probability density function; (c) Cumulated histogram (solid line: simulation, dotted line: theory) as cumulative distribution function. rate reduced from 0.0410 to 0.0022 . Fig. 10 compares the bit error rates before and after beamforming as a function of SIR for SNR = 0dB. It shows the efficacy of the beamformer, since the bit error rate is reduced.

\section{Performance comparison}

To further characterize the beamformer's

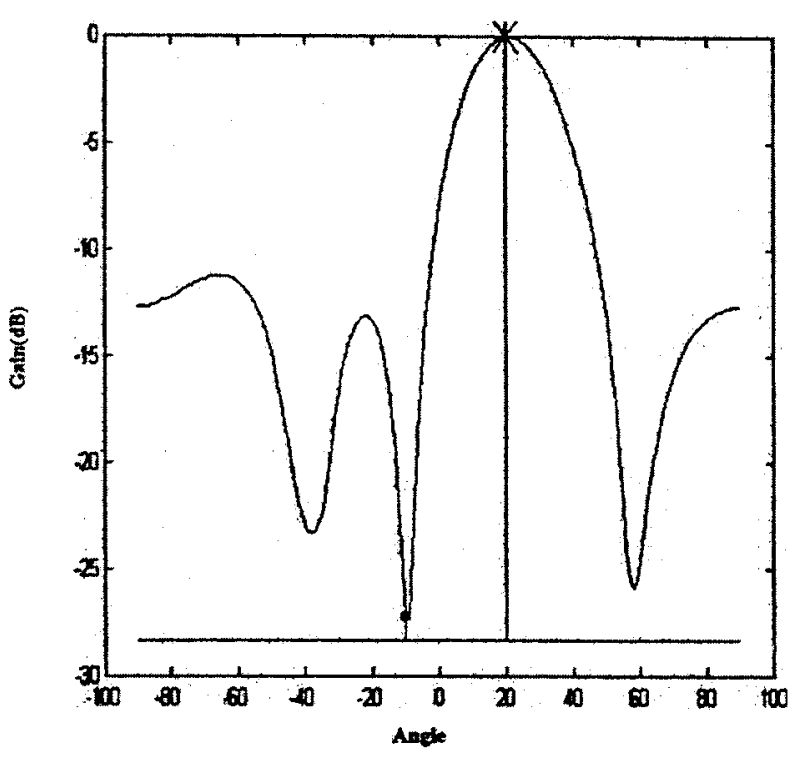

Fig. 8. Beampattern of Frost adaptive beamformer over Rician fading channel.
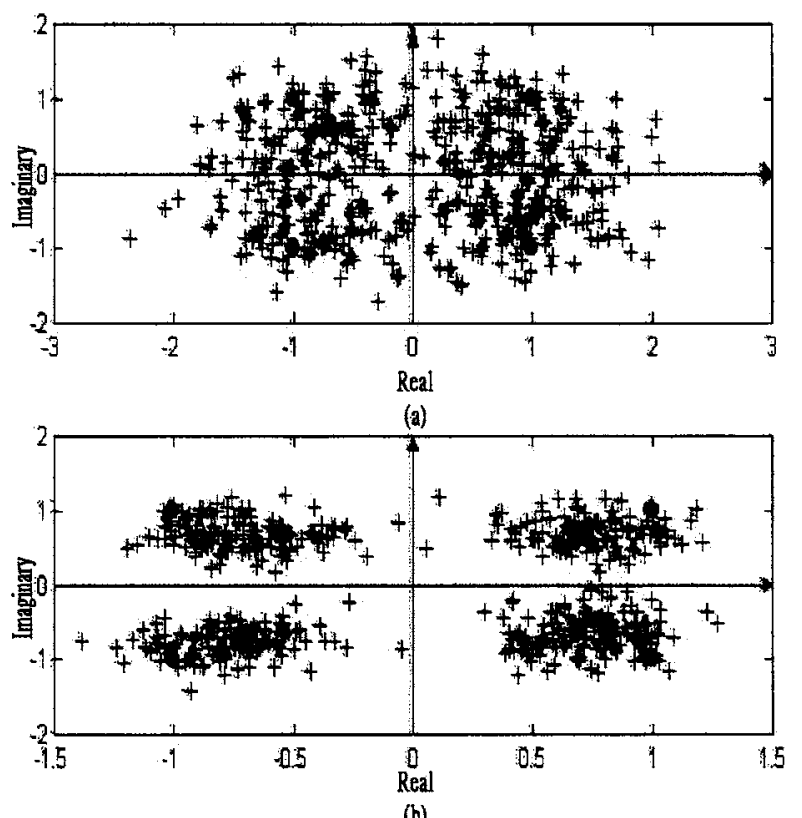

(b)

Fig. 9. Constellations of the demodulated signals, (a) before beamformer and (b) after beamformer. 
performance, two performance indices were used. They are defined as follows. The array gain is defined as the ratio of the signal-to-interference-plus-noise ratios (SINR) at the input and output of the beamformer, expressed in $\mathrm{dB}$. It is used to indicate the improvement factor, in $\mathrm{dB}$, of the SINR by means of the beamformer. The normalized mean-square error is defined as the sum of the squared difference between the beamformer output signal and the desired signal, divided by the sum of the squares of the desired signal. It is used to indicate the degrees of distortion for the beamformer output waveform, as compared to the desired signal waveform.

Fig. 11 and 12 compare, respectively, the normalized mean-square errors and the array gains of the beamformer, as a function of iterative steps, over the nonfading (ideal), Rayleigh fading, and Rician fading channels. It is anticipated that the best is the performance over nonfading channel, the second is the one over the Rician fading channel, and the worst is the one over the Rayleigh fading channel.

\section{REAL TIME IMPLEMENTATION ISSUES}

The receiver prototype system was built by using four B\&K 8104 receiving hydrophones, four B\&K 2635 charge amplifiers, TIMS low-pass filter modules, one LSI QPC C40 DSP module and one LSI QPC C40 interface module. Four receiving hydrophones, contained in a single potted stave and arranged as a linear array with a spacing of $15 \mathrm{~cm}$ (half wavelength) were

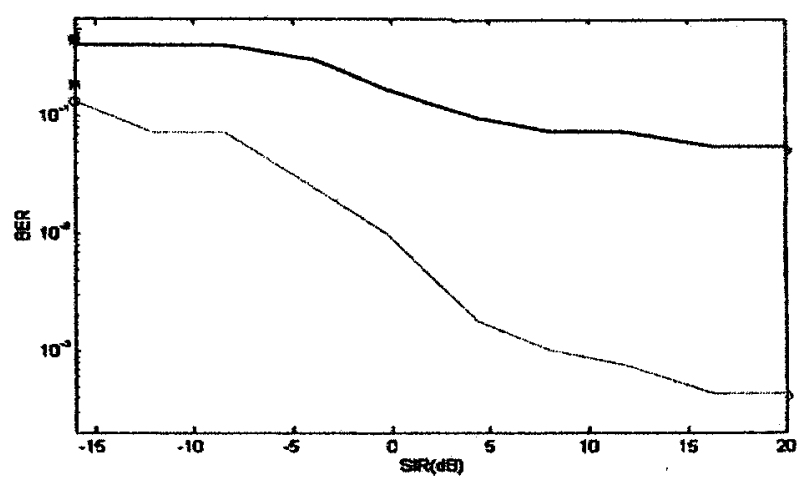

Fig. 10. Bit error rates as a function of $\mathrm{SIR}$ for $\mathrm{SNR}=0 \mathrm{~dB}$; solid line: before beamformer, dotted line: after beamformer.

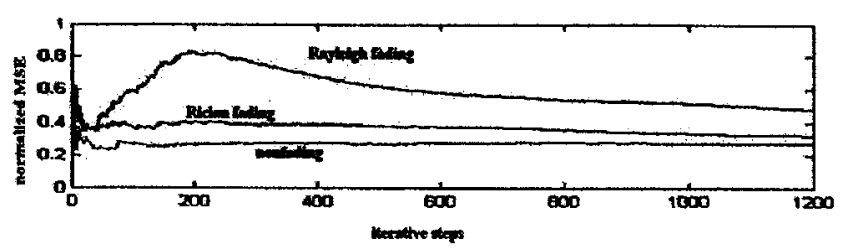

Fig. 11. Normalized mean-square errors of the Frost adaptive beamformer. deployed in an acoustic trough. The acoustic trough has $3 \mathrm{~m}$ of length, $1.5 \mathrm{~m}$ of width, and $1 \mathrm{~m}$ of height. Signals received by the hydrophone array were signal conditioned by the charge amplifiers, low-pass filtered by the anti-aliasing filters, and then fed into the ADC module for digitization purpose. The LSI QPC C40 DSP module, a product of LSI inc., has a Texas instrument TMS320C40 programmable DSP chip for performing the Frost beamformer algorithm. Compatible with the DSP module is the QPC C40 interface module which consists of an analog-to-digital converter (ADC) and a digital-to-analog converter (DAC). The ADC has a capacity of 16 channels of sample and hold for simultaneous conversion, with the fastest sampling rate of 25 $\mathrm{KHz}$ per channel and 12-bit resolution. The TIMS filter module is a 3-pole Butterworth low-pass filter with the $-3 \mathrm{~dB}$ cutoff frequency of $9 \mathrm{KHz}$ set for anti-aliasing purpose. For the Frost beamformer with four input channels and no tap per channel, it requires 43.8 microseconds of execution time before the next sample comes. Therefore, the sampling rate can not be larger than 22 . $8 \mathrm{KHz}$. After the beamforming process, the digital output is converted back to the analog form via the $\mathrm{DAC}$, and then interpolated by the TIMS low-pass filter.

The transmitter prototype system was built by using two B\&K 8105 transmitting hydrophones, two B\&K (2706 and 2713) power amplifiers, one HP function generator, one LSI C32 DSP module and one LSI C32 interface module. One transmitting hydrophone was used to transmit the BPSK signal with carrier frequency of $5 \mathrm{KHz}$ and data rate of $5 \mathrm{~K}$ bits per second as the desired signal for communication. The BPSK signal generation is described as follows. The carrier of frequency $5 \mathrm{KHz}$ was generated by a function generator, converted by the ADC in the interface module, and then mixed with an alternating binary sequence of -1 and 1 . The multiplication was done by the DSP module. Finally the mixed sequence is converted into the analog waveform via the DAC in the interface module, has its power increased by the power amplifier before fed into the transmitting hydrophone. Another transmitting hydrophone was used to transmit a sinusoid of $8 \mathrm{KHz}$ as an interference signal. The entire prototype system is depicted in Fig. 13.

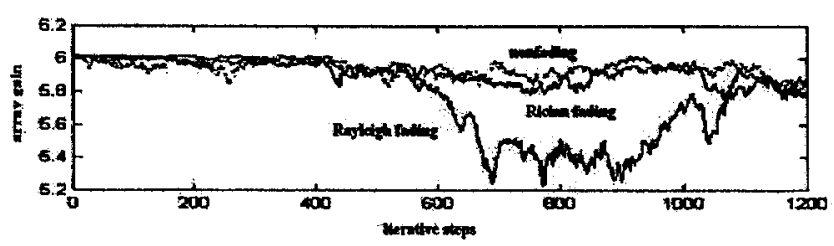

Fig. 12. Array gains of the Frost adaptive beamformer. 


\section{EXPERIMENTAL RESULTS}

Two transmitting hydrophones and four receiving hydrophones were deployed in an acoustic trough, as shown in Fig. 14. The BPSK signal described above was transmitted from 25 degree, whereas the interfering signal was transmitted from 5 degree. Both the desired and the interfering signals have the same power. The sampling rate of the receiving ADC was chosen to be 22 . $5 \mathrm{~K}$ samples per second. The step size used in Frost beamformer was set to be 0.008 . Fig. 15 shows the received waveforms of the four channels before beamforming. It is evident that the received signal in each channel is severely distorted by the interference signal and coherent signals (due to multipaths). However, the desired signal is perfectly restored after

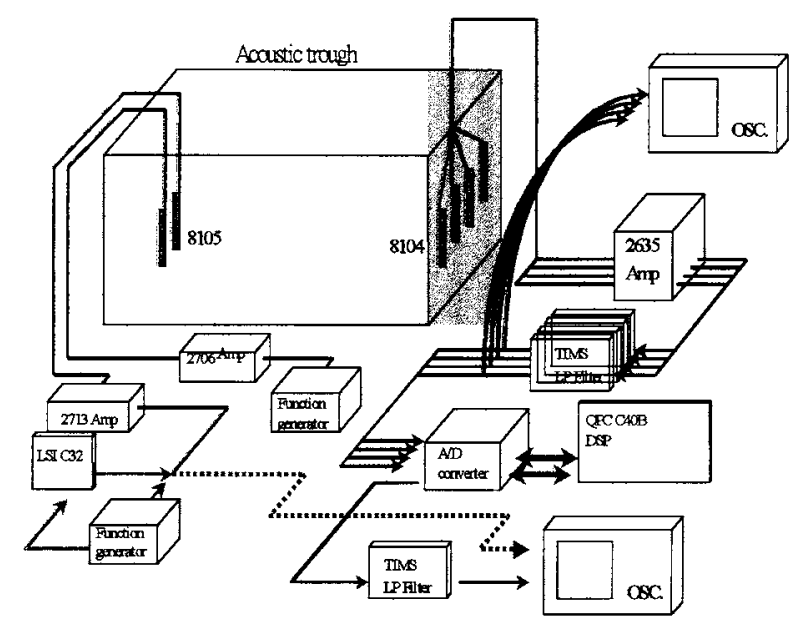

Fig. 13. Real-time beamformer prototype system.

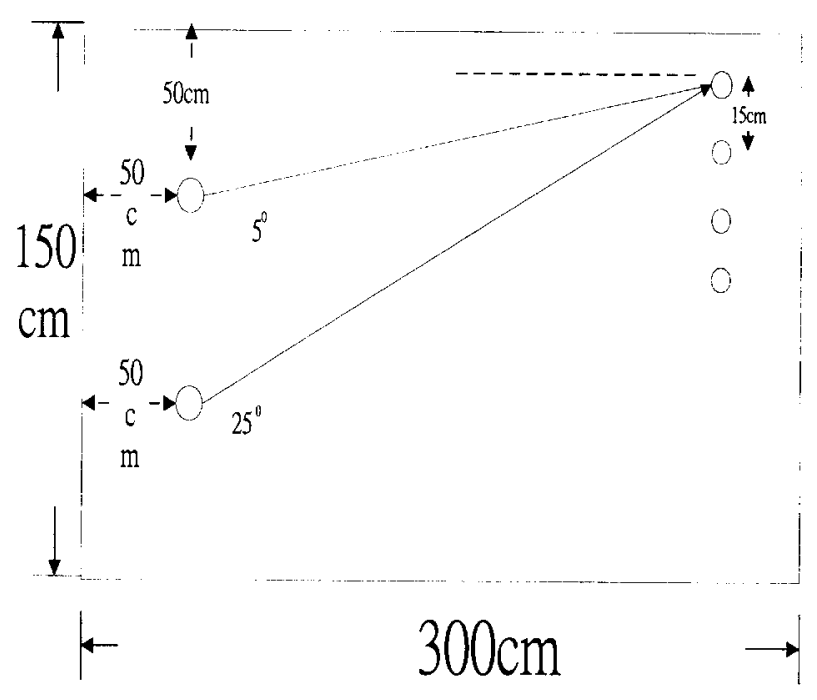

Fig. 14. Deployment of hydrophones in acoustic trough. beamforming, as depicted in Fig. 16. Although not shown in this paper, similar results can be obtained by varying the bearing angles of the interfering signal to 10 and 15 degrees.

In order to explore the cancellation problem of the desired signal with the coherent signals, only a single transmitting hydrophone was used to transmit the desired signal. By varing the bearing angle of the transmitting hydrophone, the beamformer output waveform deviates from the desired signal to some extent, as shown in Fig. 17 for 15 degree case. The coherent signals resulted from the reflections of acoustic waves tend to cancel the desired signal. This demonstrates the fact that the Frost beamformer is less effective when coherent signals due to multipaths exist [4].

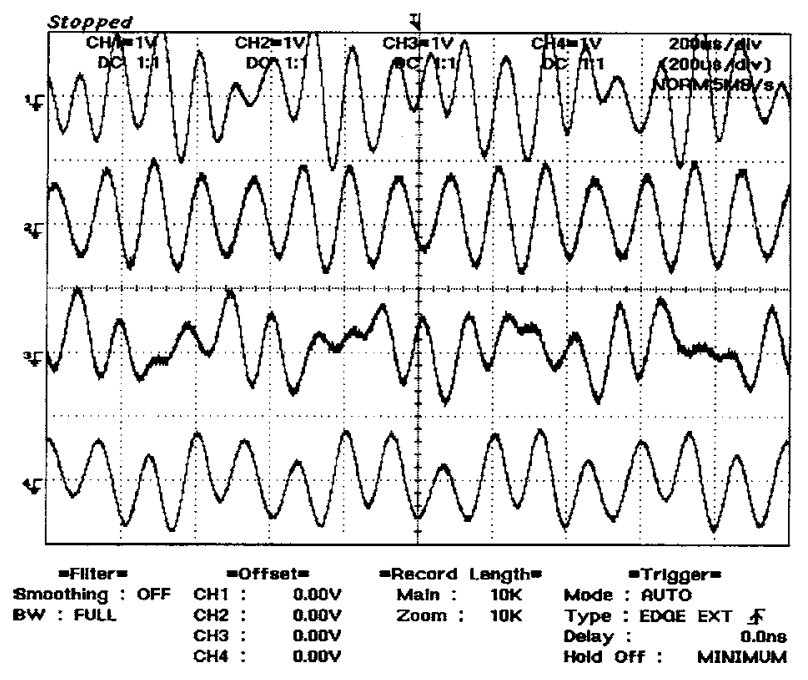

Fig. 15. The received signal waveforms at the four hydrophones before beamforming.

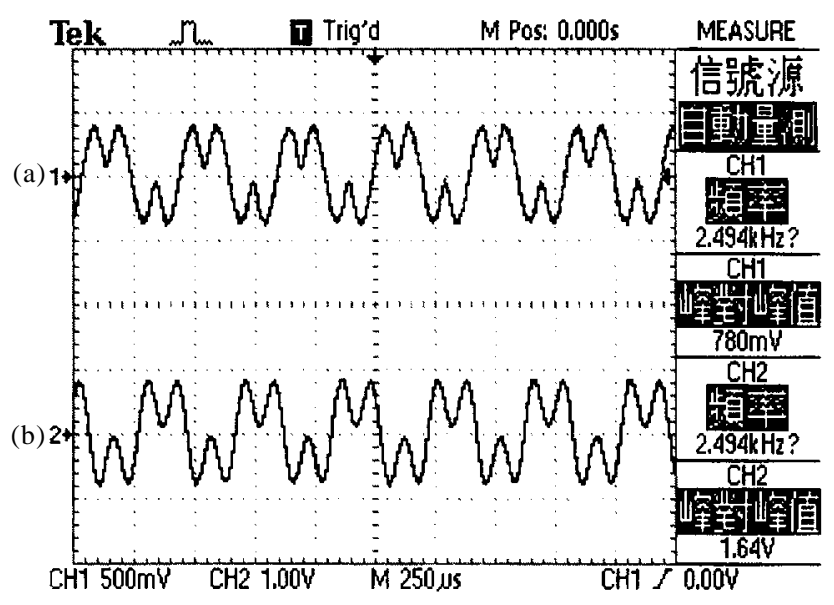

Fig. 16. Comparison of (a) desired signal and (b) beamformer output. 


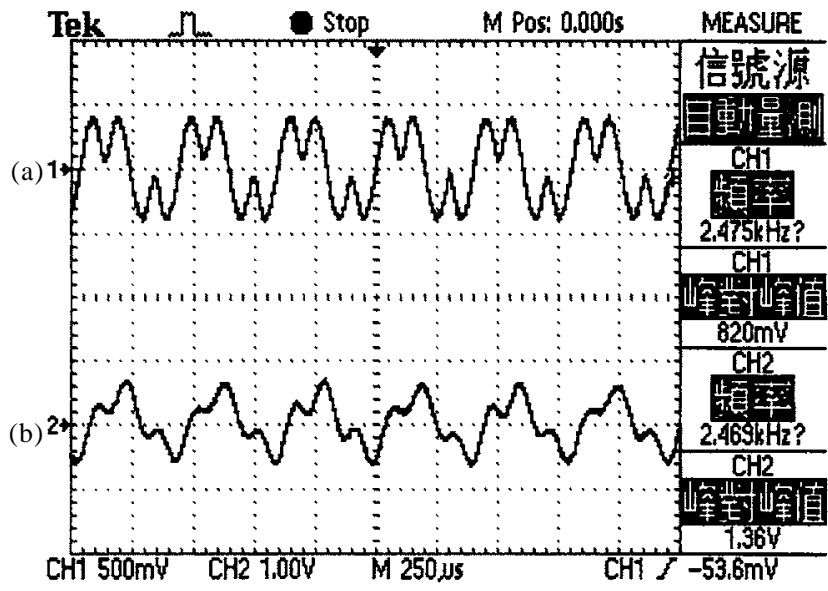

Fig. 17. Comparison of (a) desired signal and (b) beamformer output for multipath effect.

\section{CONCLUDING REMARKS}

In this paper, we have investigated the performance of Frost beamformer via computer simulations for QPSK signals transmitted over Rayleigh and Rician fading channels. Simulation results have shown that the Frost beamformer is able to improve the signalto-interference-plus-noise ratio over fading channels. As a preprocessor for underwater communications, a real-time prototype system which uses TMS320C40 DSP for the realization of Frost beamformer has been built. Through experimental work in an aconstic trough, the validity of the real-time prototype system has been verfied, and the feasibility of its use in underwater communications has been assessed.

\section{ACKNOWLEDGMENTS}

This work was supported by the National Science Council, Taiwan, R.O.C. under Contract NSC86-2611E-019-016. The authors would like to thank the National Science Council for the support of this work.

\section{REFERENCES}

1. M. Stojanovic, J.A. Catipovic, and J.G. Proakis, "Phasecoherent digital communications for underwater acoustic channels," IEEE Journal of Oceanic Engineering, Vol. 19, pp. 100-111, Jan. 1994.

2. O.R. Hinton, G.S. Howe, A.E. Adams, P.S.D. Tarbit, and B. S. Sharif, "Performance of stochastic gradient adap- tive beamformer for sub-sea acoustic communications," Proc. EUSIPCO-94 Conf., pp. 1540-1543, Edinburg, Sept. 1994.

3. J.-Y. Lee and H. Samueli, "Adaptive antenna arrays and equalization techniques for high bitrate QAM receivers, " Proceedings of ICUPC, pp. 1009-1013, Sept./Oct. 1996.

4. O.L. Frost, "An algorithm for linearly constrained adaptive array processing," Proceedings of the IEEE, Vol. 60, Aug. 1972.

5. R.T. Compton, Jr., Adaptive antenna concepts and performance, Prentice-Hall Inc., 1988.

6. L.J. Griffiths and C.W. Jim, "An alternative approach to linearly constrained adaptive beamforming," IEEE Trans. Antennas propagation, Vol. AP-30, No. 1, pp. 27-34, Jan. 1981.

7. A. Falahati, B. Woodward, and S. C. Bateman, "Underwater acoustic channel models for $4800 \mathrm{~b} / \mathrm{s}$ QPSK signals," IEEE Journal of Oceanic Engineering, Vol. 16, pp. 12-20, Jan. 1991.

8. X. Geng and A. Zielinski, "An eigenpath underwater acoustic communication channel model," Proc. OCEAN95 Conf., pp. 1189-1196, San Diego, 1995.

9. W.C. Jakes, Jr., Microwave mobile communications, J. Wiley Sons, 1974.

\section{FROST波束構成器於水下通訊之即 時實現}

$$
\text { 洪賢 昇張順雄陳玄 齡 }
$$

國立台灣海洋大學電機工程學系

$$
\text { 摘 要 }
$$

在本篇論文中，我們針對四相位移鍵 $(\mathrm{QPSK})$ 訊 號於瑞理(Rayleigh)和瑞斯(Rician)退化通道傳彪時, 經由電腦模擬以探討彿洛斯特(Frost)線性限制最小變 異適應波束構成器的特性。模擬結果顯示, Frost波束 構成器在退化通道下, 仍可以改善訊擾雜比。另外, 對於即時的水下通訊而言, 我們以TMS $320 \mathrm{C} 40$ 數位 訊號處理晶片, 來研製實現Frost波束構成器的雛形系 統。經由水槽内的實驗結果, 證實此即時波束構成器 雛形系統的正確性, 並對其應用於水下通訊作可行性 的評估。 